\title{
Impact of Galileo on Global Ionosphere Map Estimation
}

\author{
Anh Quan Le \\ (Delft University of Technology) \\ (Email: A.Q.Le@1r.tudelft.nl)
}

\begin{abstract}
The upcoming GNSS Galileo, with its new satellite geometry and frequency plan, will not only bring many benefits for navigation and positioning but also help to improve ionosphere delay estimation. This paper investigates ionosphere estimation with Galileo and compares it with the results from GPS-only and combined GPS-Galileo. The standard deviation of the Vertical Total Electron Content (VTEC) at a certain location can improve significantly by 40 per cent. Various Galileo configurations are considered to assess the differences in frequency plan and signals to be used. The IGS network, which is involved in producing the current IGS Global Ionosphere Map (GIM) is used in the simulation for more realistic results. A more accurate GIM will in its turn improve navigation and positioning performance.
\end{abstract}

\section{KEY WORDS}
1. GPS.
2. Galileo.
3. Ionosphere.
4. Design Computation.

1. INTRODUCTION. By 2010, the European GNSS Galileo will be fully operational with 27 satellites in three planes, at 56-degree inclination. Besides the benefit it brings for navigation and positioning, the new system will help to improve our understanding of the atmosphere, especially for ionosphere delay estimation. This is not just because there will be more satellites but also due to the configuration of Galileo including the satellite geometry and the frequency plan. In this study, the ionosphere estimation with Galileo will be investigated and compared with the results from GPS-only and combined GPS-Galileo to see the improvement from Galileo. Various Galileo configurations will be considered to assess the differences in frequency plan and signals to be used. To make it more realistic, the IGS station network is used in the simulation. This is the network being used to produce the current IGS Global Ionosphere Map (GIM).

\section{IONOSPHERE MODELLING.}

2.1. Observation modelling. A lot of work has been done on ionosphere estimation based on a GPS tracking network. The estimation is derived from the GPS pseudorange and carrier phase observations. Nevertheless, it holds for any other GNSS including Galileo and Glonass. If a GNSS transmits both pseudorange and carrier phase signals on two frequencies $f_{1}$ and $f_{2}$, the equations of 
observations read:

$$
\begin{aligned}
& P_{r, \mathbf{1}}^{s}=\rho_{r}^{s}+c \delta t_{r}-c \delta t^{s}+T_{r}^{s}+I_{r}^{s}+c\left(b^{s, \mathbf{1}}+b_{r, \mathbf{1}}\right)+\varepsilon_{P, \mathbf{1}} \\
& P_{r, 2}^{s}=\rho_{r}^{s}+c \delta t_{r}-c \delta t^{s}+T_{r}^{s}+\xi I_{r}^{s}+c\left(b^{s, 2}+b_{r, 2}\right)+\varepsilon_{P, \mathbf{2}} \\
& \Phi_{r, \mathbf{1}}^{s}=\rho_{r}^{s}+c \delta t_{r}-c \delta t^{s}+T_{r}^{s}-I_{r}^{s}+\nabla_{r, \mathbf{1}}^{s}+\varepsilon_{\Phi, \mathbf{1}} \\
& \Phi_{r, 2}^{s}=\rho_{r}^{s}+c \delta t_{r}-c \delta t^{s}+T_{r}^{s}-\xi I_{r}^{s}+\nabla_{r, 2}^{s}+\varepsilon_{\Phi, 2}
\end{aligned}
$$

where $P_{r, 1}^{s}, P_{r, 2}^{s}$ are the pseudorange observations on $f_{1}$ and $f_{2} . \Phi_{r, 1}^{s}, \Phi_{r, 2}^{s}$ are the carrier phase observations on $f_{1}$ and $f_{2} \cdot \rho_{r}^{s}=\left\|x^{s}-x_{r}\right\|$ is the geometric range between receiver and satellite. $x^{s}$ is the satellite position at time of transmission. $x_{r}$ is the receiver position at time of observation. $\delta t^{s}$ is the satellite clock error at time of transmission. $\delta t_{r}$ is the receiver clock error at time of observation. $T_{r}^{S}$ is the tropospheric delay. $I_{r}^{S}$ is the ionospheric delay related to the $f_{1}$ frequency. $\xi=f_{1}^{2} / f_{2}^{2}$ is the inter-frequency factor of ionospheric delay. $b^{s, 2}, b^{s, 2}$ are the satellite hardware delays. $b_{r, 1}, b_{r, 2}$ are the receiver hardware delays. $\nabla_{r, 1}^{s}, \nabla_{r, 2}^{s}$ are the ambiguities (including satellite and receiver hardware delays, in units of range). $\varepsilon_{P, 1}, \varepsilon_{P, 2}, \varepsilon_{\Phi, 1}, \varepsilon_{\Phi, 1}$ are unmodelled errors (such as noise and multipath). And $c$ is the speed of light in vacuum.

Taking the geometry free linear combination:

$$
\begin{aligned}
& P_{r, 4}^{s}=P_{r, \mathbf{1}}^{s}-P_{r, \mathbf{2}}^{s} \\
& \Phi_{r, \mathbf{4}}^{s}=\Phi_{r, \mathbf{1}}^{s}-\Phi_{r, \mathbf{2}}^{s}
\end{aligned}
$$

it can be seen that all the frequency-independent elements are eliminated including geometric range, satellite and receiver clock errors, and tropospheric delay:

$$
\begin{aligned}
& P_{r, 4}^{S}=+\xi_{4} I_{r}^{S}+c\left(\Delta b^{S}-\Delta b_{r}\right)+\varepsilon_{P, 4} \\
& \Phi_{r, 4}^{S}=-\xi_{4} I_{r}^{S}+\nabla_{r, 4}^{s}+\varepsilon_{\Phi, 4}
\end{aligned}
$$

where

$\xi_{4}=1-\xi=1-f_{1}^{2} / f_{2}^{2}$ is the geometry free linear combination factor

$\Delta b^{s}=b^{s, 1}-b^{s, 2}$ is the differential satellite hardware bias (between frequencies)

$\Delta b_{r}=b_{r, 1}-b_{r, 2}$ is the differential receiver hardware bias (between frequencies)

$\nabla_{r, 4}^{s}=\nabla_{r, 1}^{s}-\nabla_{r, 2}^{s}$ is the ambiguity of the geometry free linear combination

The ionosphere range delay is caused by free electrons along the transmission path, the amount is usually called total electron content (TEC). They are related by equation (4) [Parkinson et al, 1996].

$$
I_{r}^{s}=40 \cdot 3 \frac{E(z)}{f^{2}}
$$

with $I_{r}^{S}$ the range delay $[\mathrm{m}], z$ the zenith angle, $E(z)$ the total electron content (TEC) along the path [TECU] and $f$ the frequency $[\mathrm{Hz}]$.

In the ionosphere map, the vertical value of the TEC is needed instead of the line-of-sight TEC value. The line-of-sight TEC can be mapped into vertical TEC using a single-layer model with a mapping function:

$$
E(z)=F(z) E_{v}
$$

where $E(z), E_{v}$ are the slant and vertical TEC values, and $F(z)$ is the mapping function. 
The single-layer model mapping function is given in [Schaer, 1999] as:

$$
F(z)=\frac{1}{\cos z^{\prime}} \quad \text { with } \quad \sin z^{\prime}=\frac{R}{R+H} \sin z
$$

with

$z^{\prime}$ the satellite's zenith angle at the ionospheric pierce point

$H$ the height of the single layer

and $\mathrm{R} \approx 6371 \mathrm{~km}$ the mean Earth radius

More precisely, the mapping function can be modified to be:

$$
F(z)=\frac{1}{\cos z^{\prime}} \quad \text { with } \quad \sin z^{\prime}=\frac{R}{R+H} \sin (\alpha z)
$$

Usually, the single layer height $H$ is assumed to be about $450 \mathrm{~km}$. However, it is different in the modified single-layer model mapping function. It is, with $H=506.7 \mathrm{~km}$ and $\alpha=0.9782$, shown to better fit the JPL extended slab model than the original single-layer model mapping function (see [Schaer, 1999] and [CODE, 2004]). From (4) and (5), it gives:

$$
I_{r}^{S}=40 \cdot 3 F(z) \frac{E_{v}}{f^{2}}
$$

Replacing (8) into (3), we obtain:

$$
\begin{gathered}
P_{r, 4}^{s}=+40 \cdot 3 F(z) \frac{f_{2}^{2}-f_{1}^{2}}{f_{1}^{2} f_{2}^{2}} E_{v}+c\left(\Delta b^{s}-\Delta b_{r}\right)+\varepsilon_{P, 4} \\
\Phi_{r, 4}^{s}=-40 \cdot 3 F(z) \frac{f_{2}^{2}-f_{1}^{2}}{f_{1}^{2} f_{2}^{2}} E_{v}+\nabla_{r, 4}^{s}+\varepsilon_{\Phi, 4}
\end{gathered}
$$

with $E_{v}$ the vertical TEC value at the ionospheric pierce point.

2.2. Ionosphere parameterisation. In order to derive regional or global ionosphere maps, a model or a TEC representation will be needed. There are several parameterisation methods to represent the vertical TEC values as mentioned in [Schaer, 1999] and [Gao, 2004]. Among those, the simplest method is to deploy a two dimensional Taylor expansion and then estimate the coefficients. Other more sophisticated methods include the spherical harmonic ( $\mathrm{SH}$ ) expansion developed by CODE and implemented in the Bernese GPS software, using the normalised associated Legendre function. JPL (Jet Propulsion Laboratory) also use the SH expansion but with an almost uniform grid whereas ESA (European Space Agency), EMR (Energy, Mines and Resources), UPC (Polytechnical University of Catalonia), and UNB (University of New Brunswick) use their own models to describe the TEC representation.

In this design study, because no real data is involved, a simple two dimensional Taylor expansion is used:

$$
E_{v}(\beta, s)=\sum_{n=0}^{n_{\max }} \sum_{m=0}^{m_{\max }} E_{n m}\left(\beta-\beta_{0}\right)^{n}\left(s-s_{0}\right)^{m}
$$

where

$(\beta, s)$ are the solar-geographic coordinates of the ionospheric pierce point 
$n_{\max }, m_{\max }$ are the maximum orders of the two dimensional Taylor series expansion in latitude and longitude

$E_{n m}$ are the unknown coefficients of the Taylor series expansion

$\left(\beta_{0}, s_{0}\right)$ are the solar-geographic coordinates of the origin of the expansion

Note that this parameterisation method is not suitable for global ionosphere mapping due to its characteristics but it is rather simple and can be used for regional ionosphere modelling [Schaer, 1999]. For large regional ionosphere mapping, the orders of Taylor expansion can be as large as 10 . Whereas, orders of six are suitable for local or small regional ionosphere mapping, resulting in 36 unknown parameters and will be used in this study $\left(n_{\max }=m_{\max }=5\right)$.

2.3. Hardware delays. Due to the fact that hardware delays are frequency dependent in both satellite and receiver, they are not cancelled in the geometry free linear combinations and the absolute values cannot be determined because the (satellite or receiver) hardware delay may be shifted by an undistinguishable common bias from the (satellite or receiver) clock error. The relative values between frequencies (in both satellite and receiver) are still estimable and form the name Differential Code Biases (DCB) [CODE, 2004]. Although they only change slowly with time and can be considered constant, as side products of ionosphere maps, they can be included in the model and estimated as well. It will increase the number of unknown parameters by the number of satellites (24 in case of GPS and 27 in case of Galileo) and the number of stations in use. However, there will be a rank defect of 1 in the design matrix that makes it impossible to estimate all satellites and receivers DCBs at the same time. We will see it in the following example of code measurement. Assuming that $m$ receivers are observing $n$ satellites simultaneously, the system of observation equations for one single epoch reads:

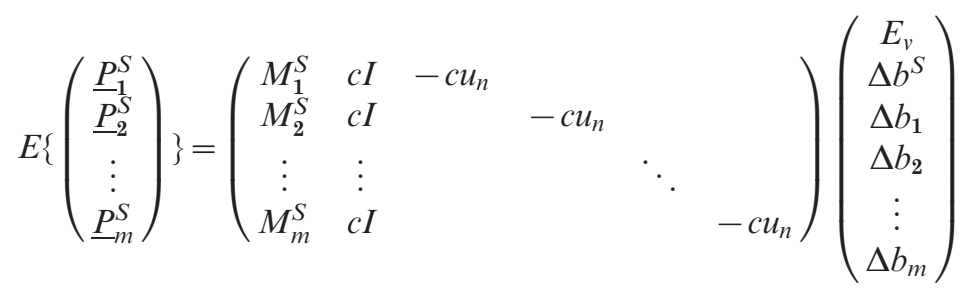

where

$\underline{P}_{r}^{S}(n \times 1)$ is the observation set from receiver $r$ to all satellites $(r=1 . . m)$, each of them is a geometry-free linear combination of code observations as in (3)

$M_{r}^{s}$ is the mapping from slant to vertical TEC at receiver $r$ (as in (9)), then to the Taylor expansion coefficients (10)

$I(n \times 1)$ is the identity matrix

$u_{n}(n \times 1)$ is the column vector with all element equal to 1

$E_{v}$ are the unknown Taylor expansion coefficients

$\Delta b^{s}(n \times 1)$ is the set of all satellites' DCBs

$\Delta b_{r}$ is the DCB of receiver $r$

$c$ is the speed of light

If we denote the design matrix by $A$ and a column vector $v$ as:

$$
v=\left(\begin{array}{lll}
0 & u_{n}^{T} & u_{m}^{T}
\end{array}\right)^{T}
$$


then obviously, $A v=0$. Therefore the design matrix $A$ is rank deficient and some constraint must be deployed to avoid this problem, for instance, fixing one DCB, of either a satellite or a receiver.

If receiver hardware delays are estimated, a large number of stations will be needed to get more redundancy or more sequential epochs should be combined. With high grade receivers, they only change slowly and can be considered constant in a day. [Hernández-Pajares, 2004] has shown that the receiver DCBs are quite stable for most of the IGS stations, within 1 ns margin in 60 days. Hence, fixing all the receiver DCBs will help both avoiding rank deficiency and improving redundancy.

In the phase measurement, the hardware delays cannot be distinguished from the ambiguity (see (9)) and each pair satellite-receiver will have one ambiguity to be resolved. Therefore, more than one epoch are needed to deal with the ambiguity problem. The rank deficiency also occurs here. In this design study, however, the ambiguities are assumed to be fixed and solved. Hence, the phase observation equation is reversed to the same as the code observation equation (of course, except for the measurement precision that differs roughly with a factor of 100 ).

2.4. Stochastic modelling. To estimate the unknown parameters of the ionosphere map with the above functional model, a stochastic model (of the observations) is needed. Assuming no correlation between any pair of measurements, pseudorange or carrier phase (which is quite realistic), the variances of the geometry-free linear combinations (2) read:

$$
\begin{aligned}
& Q_{P_{4}}=Q_{P_{1}}+Q_{P_{2}} \\
& Q_{\Phi_{4}}=Q_{\Phi_{1}}+Q_{\Phi_{2}}
\end{aligned}
$$

with

$Q_{P_{1}}, Q_{P_{2}}$ and $Q_{P_{4}}$ the variances of pseudorange measurements on $f_{1}, f_{2}$ and geometry-free linear combination respectively

$Q_{\Phi_{1}}, Q_{\Phi_{2}}$ and $Q_{\Phi_{4}}$ the variance of carrier phase measurements

The measurement noise magnitude depends on various elements including frequency (of carrier or chip rate of code) and elevation angle to satellite. Based on current experience, in the case of GPS, the precision (standard deviation) of code and phase at zenith can be assumed as 30 centimetres and 3 millimetres, respectively. With Galileo, the carrier phase frequencies are quite close to those of GPS and hence the precision of the phase. The code measurements, however, are modulated with different chip rates for different signals as stated in [Hein et al, 2003] and can have better precision. Nevertheless, in this design study, it is reasonable to assume the code measurement noise is equal for both systems and takes the (rather conservative) value of 30 centimetres at zenith.

For satellites at any elevation angles other than at zenith, the observations are de-weighted. The variances are related as:

$$
Q(z)=\frac{Q_{0}}{\cos ^{2} z}
$$

with $z$ the zenith angle, $Q_{0}$ and $Q(z)$ the variances at zenith $(z=0)$ and at the zenith angle $z$, respectively. 


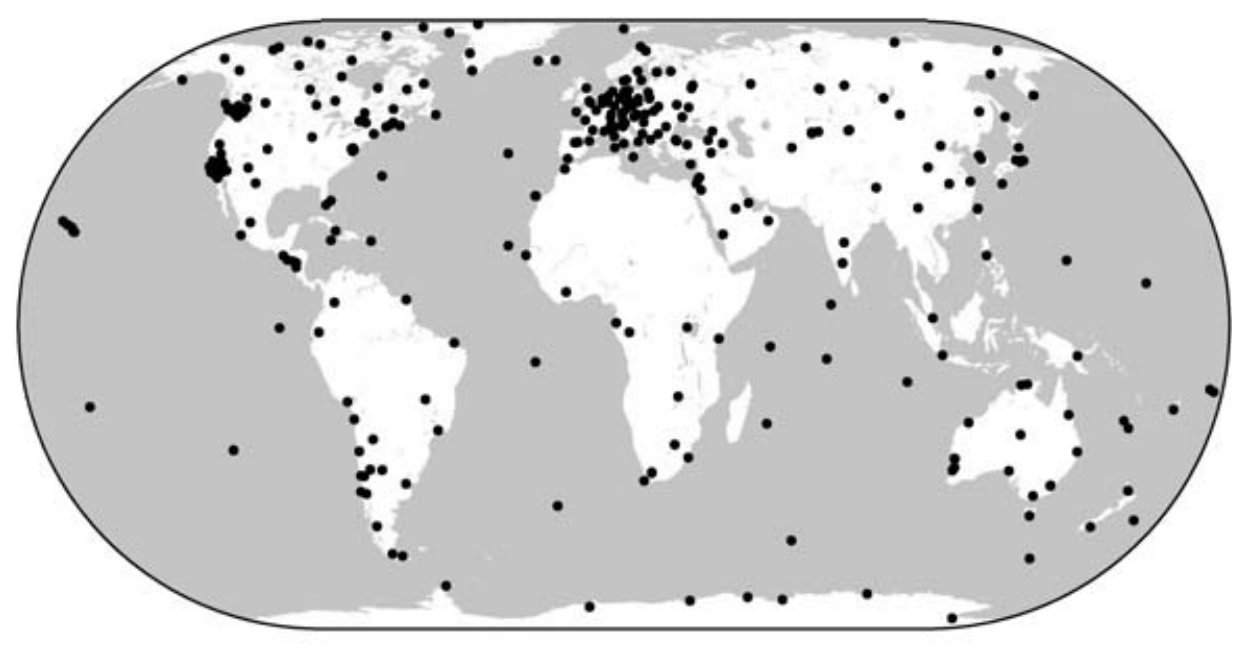

GMIII Aug 917206020004

Figure 1. IGS network distribution, graph from [IGS, 2004].

Summarising, the variances of the geometry-free linear combinations in (13) can be rewritten as:

$$
\begin{aligned}
Q_{P_{4}}(z) & =\frac{0 \cdot 18}{\cos ^{2} z}\left[\mathrm{~m}^{2}\right] \\
Q_{\Phi_{4}}(z) & =\frac{18}{\cos ^{2} z}\left[\mathrm{~mm}^{2}\right]
\end{aligned}
$$

3. PERFORMANCE EVALUATION. All the IGS stations [IGS, 2004] are used in this design study to estimate the ionosphere. Most of them are situated in North America and in Europe; nevertheless, the network is dense enough for ionosphere estimation, see Figure 1. By the unequal distribution of the network, the impact of network densification on ionosphere estimation also can be seen later in the results.

For the current GPS, signals on only two frequencies are being transmitted:

$$
\begin{aligned}
& L 1=1,575 \cdot 42 \mathrm{MHz} \\
& L 2=1,227 \cdot 60 \mathrm{MHz}
\end{aligned}
$$

According to [Hein et al, 2003], the Galileo frequencies are designed to be:

$$
\begin{aligned}
L 1 & =1,575.42 \mathrm{MHz} \\
E 5 A & =1,176.45 \mathrm{MHz} \\
E 5 B & =1,207 \cdot 14 \mathrm{MHz} \\
E 6 & =1,278.75 \mathrm{MHz}
\end{aligned}
$$

Among those, the frequency-couples to be used for dual-frequency receivers are: $L 1-E 5 A, L 1-E 5 B$ and $L 1-E 6$. In order to assess the effect of different frequencies, one 
Table 1. GPS and Galileo nominal constellations.

\begin{tabular}{lll}
\hline & \multicolumn{1}{c}{ GPS } & Galileo \\
\hline Constellation (sats/planes) & $24 / 6$ & $27 / 3$ \\
Semi-major Axis (km) & $26561 \cdot 750$ & $29600 \cdot 318$ \\
Orbital Inclination (deg) & 55 & 56 \\
Repeat cycle (days/orbits) & $1 / 2$ & $10 / 17$ \\
\hline
\end{tabular}

lowest frequency and one highest frequency are chosen, namely E5 $A$ and E6, respectively (each time combined with $L 1$ ).

In the simulation, satellite positions are computed from a Yuma almanac with the nominal constellations of both GPS and Galileo. An overview is given in Table 1. The Galileo satellite orbits are said to be finalised, according to [Zandbergen et al, 2004]. TEC maps are produced for one day with 5-minute sampling interval for the whole Earth, creating 289 maps. However, due to the characteristics of the Taylor expansion, only part of the maps will be used in the comparison, within 60 degrees from the origin, for both latitude and longitude. With the Taylor expansion, only relative latitude and longitude (to the origin of the expansion) are of interest, it is not important whether a geographic or a solar-geographic system is used. For the sake of simplicity, geographic latitude and longitude are used.

Although the receiver differential code biases (DCBs) can be estimated as well along with other parameters, it is assumed that they are known (e.g. from a calibration) to have more redundancy since every epoch is processed separately and independently in this study. The satellite DCBs change slowly but are necessary to be estimated. Two cases will be considered, one with the satellite DCBs included as unknown parameters and the other considering them known.

The unknown parameters, including the coefficients of the Taylor expansion in (10) and the satellite DCBs, are estimated once every epoch. Then they are propagated to vertical TEC values of a grid with $1^{\circ} \times 1^{\circ}$ resolution, from $-60^{\circ}$ to $+60^{\circ}$ both latitude and longitude. Note that, in this design study, only precision is concerned, meaning that real estimates of the parameters are not computed but the standard deviations of the parameters' estimators.

Beside all the above mentioned configurations to be processed, two more cases will be considered. They are the cases in which measurements on the $E 5 \mathrm{~A}$ frequency of Galileo are assumed to have higher precision, say $10 \mathrm{~cm}$ (standard deviation). The Galileo-only and GPS-Galileo combined configurations will be taken into account in order to see the best achievable performance. These cases will be marked with asterisks to be distinguished from the others.

3.1. Regional maps. The formal accuracy (standard deviation) of vertical TEC is shown in Figures 2 (minimum maps) and 3 (maximum maps) with the summary in table 2 . Note that only graphs of the without-DCB-estimation cases are shown. The other cases give relatively similar results with poorer accuracies. For each configuration, two graphs, minimum and maximum per location over 24 hours, are shown. In cases without (satellite) DCB estimation, the number of unknown parameters is equal to the number of the Taylor expansion's coefficients, which is 36 . When DCBs are included as unknowns, it is added by the number of satellites. Hence, it is 60, 63 and 87 for GPS-only, Galileo-only and the combined case respectively. 


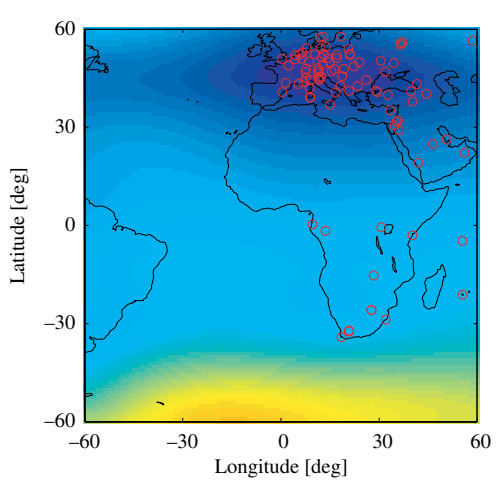

(a) GPS only (L1-L2)

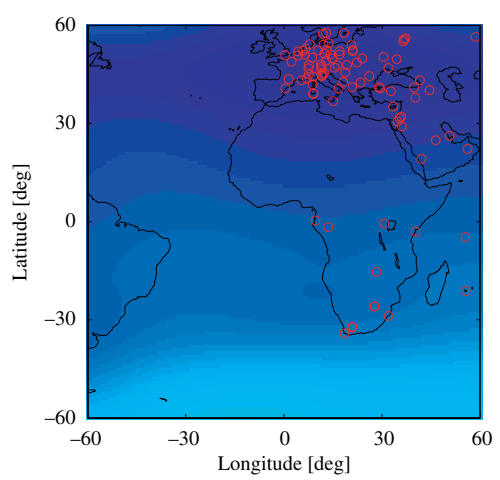

(c) GAL only (L1-E5A*)

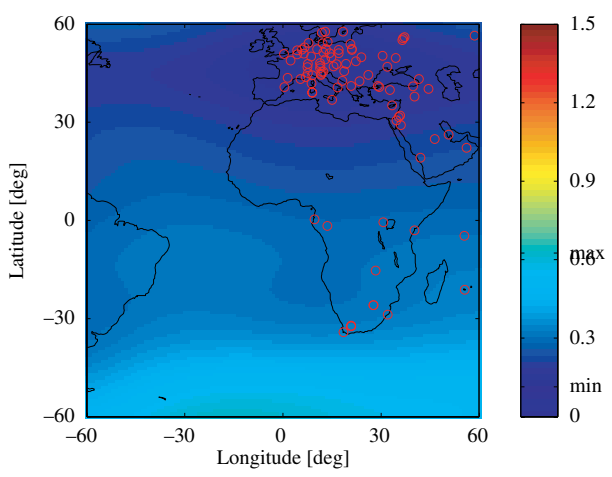

(e) GPS-GAL (L1-L2-E5A)

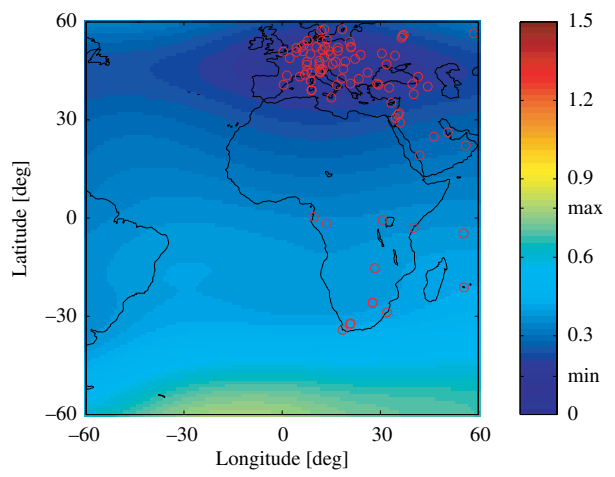

(b) GAL only (L1-E5A)

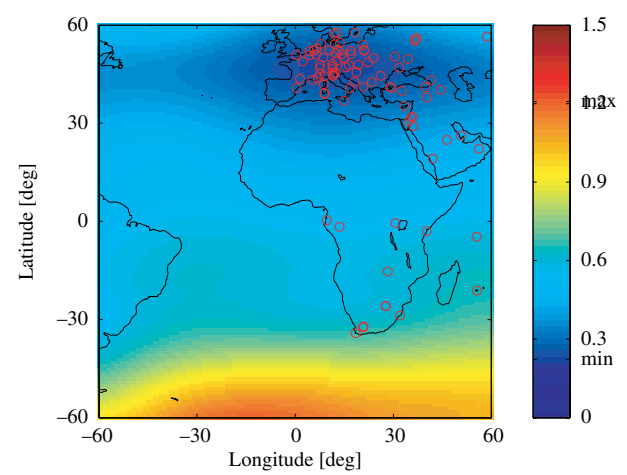

(d) GAL only (L1-E6)

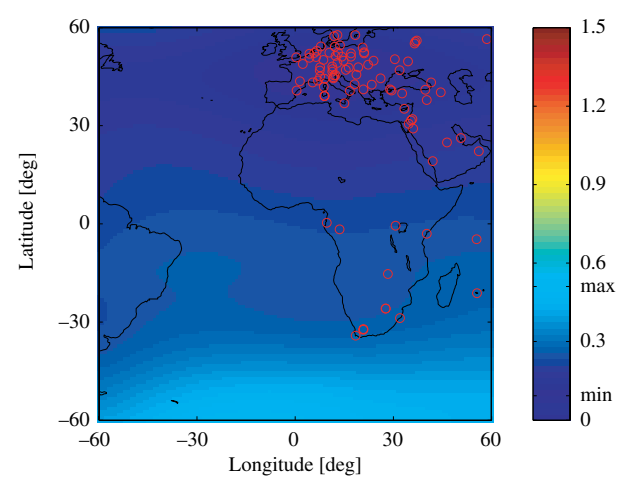

(f) GPS-GAL (L1-L2-E5A*)

Figure 2. Minimum standard deviation of vertical TEC for different configurations without DCB estimation [TECU]. Minimum per location, over full 24-hour period. The circles represent the IGS stations.

It can be seen from the results that the most effective factor on ionosphere estimation is the frequency choice. $E 6$, the frequency closest to $L 1$, gives the worst result though the Galileo satellite geometry is somewhat better than GPS, at least in the studied region. The satellite DCBs accuracy is not improved much even in the combined configurations. The high precision Galileo-only shows its great potential of 


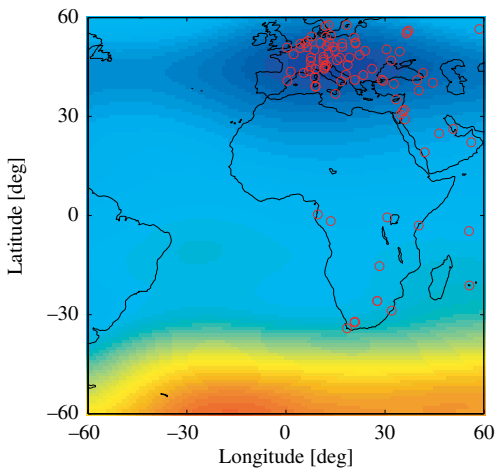

(a) GPS only (L1-L2)

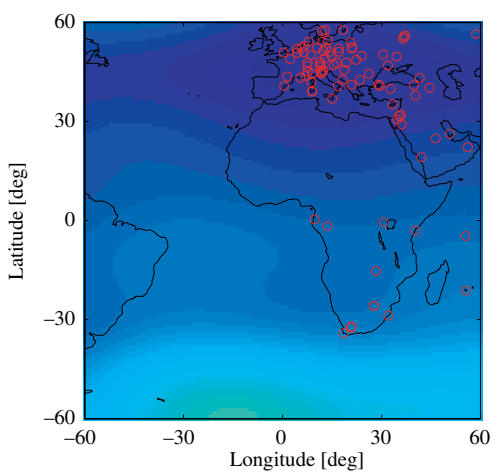

(c) GAL only (L1-E5A*)

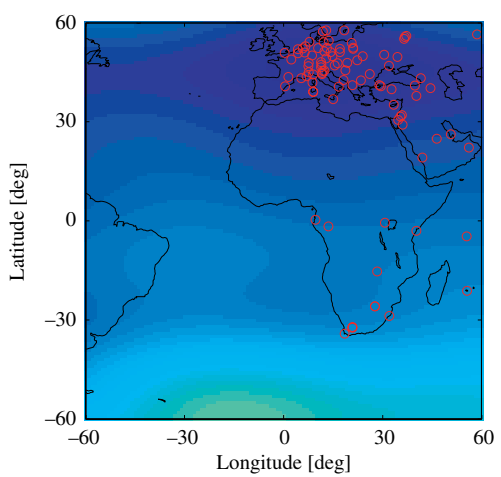

(e) GPS-GAL (L1-L2-E5A)
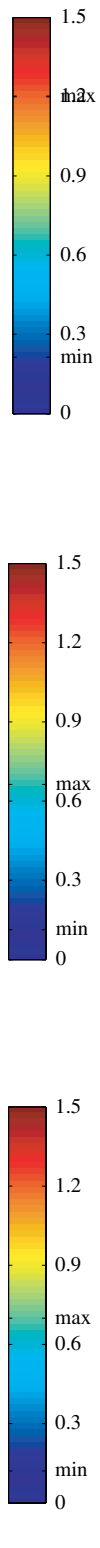

Figure 3. Maximum standard deviation of vertical TEC for different configurations without DCB estimation [TECU]. Maximum per location, over full 24-hour period. The circles represent the IGS stations.

better performance, even in comparison with the normal combined cases. If the $L 1$ precision can also be that good, it will improve the overall accuracy significantly. The inclusion of satellite DCB estimation reduces the ionosphere estimation accuracy; the standard deviation increases by about $0 \cdot 3-0 \cdot 4$ TECU. However, note that the satellite DCB set in this case is estimated at every epoch whereas it is more realistic to estimate 
Table 2. Formal accuracy (standard deviation) of estimated vertical TEC values [TECU] and DCBs [ns] (asterisks imply the high precision cases, $\sigma=0 \cdot 1 \mathrm{~m}$ instead of default $\sigma=0 \cdot 3 \mathrm{~m}$ ). Note that $1 \mathrm{TECU}=0 \cdot 162 \mathrm{~m}$ on $\mathrm{L} 1$ and $1 \mathrm{~ns}=0.3 \mathrm{~m}$. Minimum and maximum values are given, taken over all locations $\left(E_{v}\right)$ and over the full 24-hour timespan (both $E_{v}$ and DCB).

\begin{tabular}{|c|c|c|c|c|c|}
\hline & Configuration & $\mathrm{E}_{\mathrm{v}} \min$ & $\mathrm{E}_{\mathrm{v}} \max$ & $\mathrm{DCB} \min$ & DCB max \\
\hline \multirow[t]{6}{*}{ No DCB est. } & GPS only (L1-L2) & $0 \cdot 18109$ & $1 \cdot 20230$ & - & - \\
\hline & GAL only (L1-E5A) & $0 \cdot 14447$ & $0 \cdot 88929$ & - & - \\
\hline & GAL only (L1-E5A*) & $0 \cdot 10768$ & $0 \cdot 66284$ & - & - \\
\hline & GAL only (L1-E6) & $0 \cdot 22132$ & $1 \cdot 3623$ & - & - \\
\hline & GPS-GAL (L1-L2-E5A) & $0 \cdot 11437$ & $0 \cdot 70018$ & - & - \\
\hline & GPS-GAL (L1-L2-E5A*) & $0 \cdot 09354$ & $0 \cdot 57171$ & - & - \\
\hline \multirow[t]{6}{*}{ With DCB est. } & GPS only (L1-L2) & $0 \cdot 52019$ & $1 \cdot 66110$ & $0 \cdot 23820$ & $0 \cdot 68486$ \\
\hline & GAL only (L1-E5A) & $0 \cdot 37779$ & $1 \cdot 16910$ & $0 \cdot 22700$ & $0 \cdot 61067$ \\
\hline & GAL only (L1-E5A*) & $0 \cdot 28159$ & $0 \cdot 87141$ & $0 \cdot 16919$ & $0 \cdot 45518$ \\
\hline & GAL only (L1-E6) & $0 \cdot 57875$ & 1.79100 & $0 \cdot 22700$ & $0 \cdot 61067$ \\
\hline & GPS-GAL (L1-L2-E5A) & $0 \cdot 30798$ & $0 \cdot 92921$ & $0 \cdot 17740$ & $0 \cdot 55024$ \\
\hline & GPS-GAL (L1-L2-E5A*) & $0 \cdot 24881$ & 0.74935 & $0 \cdot 15540$ & $0 \cdot 51372$ \\
\hline
\end{tabular}

it once in 24 hours, knowing its nature of slow change. It would then give not very different results from the cases without DCB estimation.

3.2. Time series. In addition, time series of the standard deviation of the vertical TEC from the estimated ionosphere maps at certain locations are plotted in Figure 4. The locations chosen are the four corner points of the regional maps, namely at \pm 60 degree both latitude and longitude, and the origin of the maps. Different estimation configurations (only without DCB estimation) are presented, showing the variability over time. The effects of different satellite geometries (GPS, Galileo and GPS-Galileo combined) as well as the network geometry (or density) on the estimation can be seen here; the variation with time is related to satellite geometry and the magnitude is caused partly by the station network. In all cases, the accuracy is not so good at -60 latitude (the green and blue lines) due to the number of stations around (see Figure 1). Besides, the accuracy variation is also large there. They, the accuracy and its variation, are more or less the same for latitude 0 and +60 . Moreover, the impact of DCB estimation can be seen clearly in the last time series plot with the increment of about 0·3-0.4 TECU (Figure 5, p 292).

4. CONCLUSIONS. Through all the results obtained in the study, the big difference in the two Galileo-only configurations, L1-E5A and L1-E6, can be seen. It demonstrates the importance of frequency choice, the key factor in ionosphere estimation. The inclusion of satellite DCBs in the estimation at every epoch has significant effect on precision of vertical TEC since the number of unknowns is doubled. However, if they are considered constant but unknown and only estimated once in the whole time span (e.g. 24 hours), the effect will be negligible as the number of redundancy does not change very much.

It is obviously the serious improvement in going from current GPS-only ( $L 1-L 2)$ to GPS-Galileo combined, with any of those Galileo configurations. However, the improvement from Galileo-only $(L 1-E 5 A)$ to the combined case is rather small implying that Galileo (L1-E5A) alone performs well and clearly better than GPS (L1-L2) 
alone. Especially, in the case of high precision measurement Galileo-only (which will likely be the real Galileo configuration), the performance is even better than in the combined case with current GPS and Galileo with the same precision assumption. It is not so surprising since the modulation scheme is much more advanced in Galileo with larger tracking bandwidth though it may also result in signal acquisition difficulty.

The carrier phase measurement is omitted from all the results shown. However, based on the relative precision between code and phase, the same analysis can be made for carrier phase results [Schaer, 1999] though the ambiguity resolution will slightly reduce the accuracy.

\section{REFERENCES}

CODE website (2004). Center for Orbit Determination in Europe, Astronomy Institute (AIUB), University of Berne. http://www.aiub.unibe.ch/ionosphere.html

Hein, G. W., J. Godet, J. Issler, J. Martin, P. Erhard, R. Lucas-Rodriguez and T. Pratt (2003). Galileo Frequency and Signal Design. GPS World, June, 30-37.

Hernández-Pajares, M. (2004). Performance of IGS Ionosphere TEC Maps, Status Report of IGS Iono WG. Paper presented at Celebrating a Decade of the International GPS Service, IGS Workshop, Bern, Switzerland. March 1-5, 2004.

IGS website (2004). International GPS Service. http://igscb.jpl.nasa.gov/

Liu, Z. and Y. Gao (2004). Ionospheric TEC predictions over a local area GPS reference network. GPS Solutions, 8, 1, 23-29.

Liu, X., C. Tiberius and C. D. de Jong (2004). Modelling of differential single difference receiver clock bias for precise positioning. GPS Solutions, 7, 4, 209-221.

Parkinson, B. W. and J. J. Spiker (eds) (1996). Global Positioning System: theory and applications (Vol. 1). Progress in Astronautics and Aerodynamics. Vol. 163. AIAA. Washington.

Schaer, S. (1999). Mapping and Predicting the Earth's Ionosphere using the Global Positioning System. $\mathrm{Ph} . \mathrm{D}$ thesis. AIUB, University of Berne, Berne.

Zandbergen, R., S. Dinwiddy, J. Hahn, E. Breeuwer and D. Blonski (2004). Galileo Orbit Selection. Proceedings of ION GNSS2004, Long Beach CA, 1881-1892. 


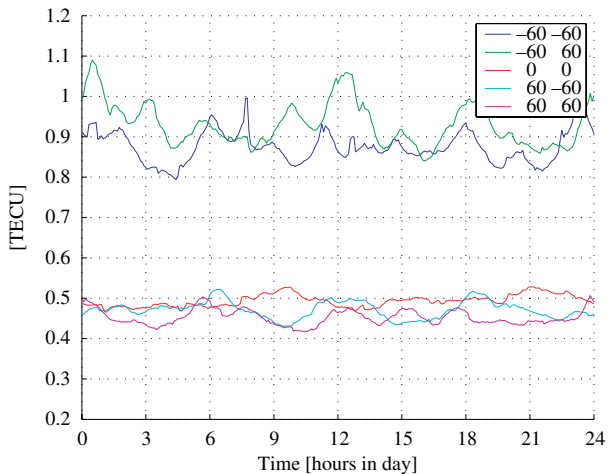

(a) GPS only (L1-L2)

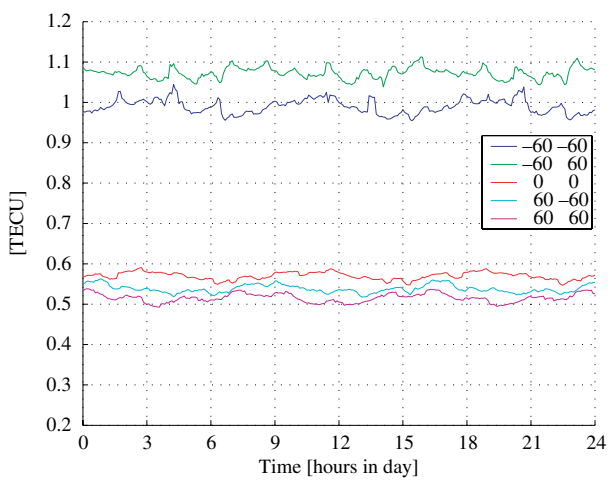

(c) GAL only (L1-E6)

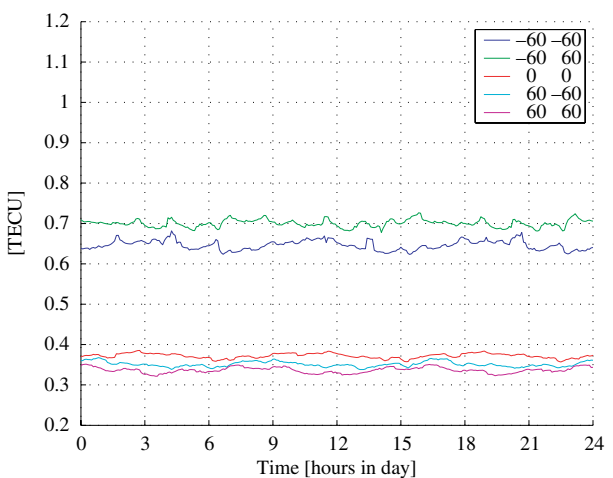

(b) GAL only (L1-E5A)

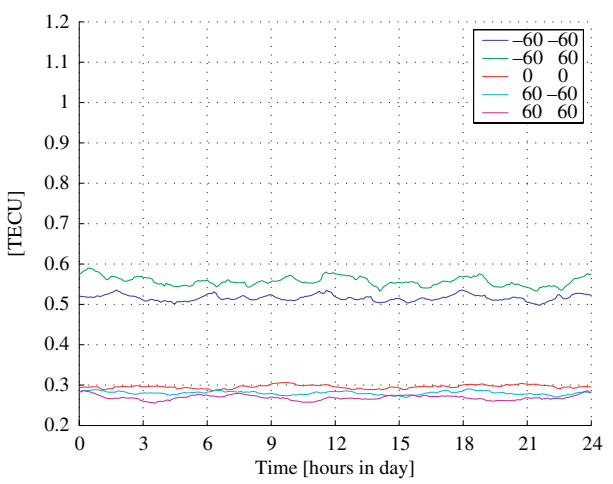

(d) GPS-GAL (L1-L2-E5A)

Figure 4. Time series of the standard deviation of the vertical TEC at the corners of the area and in the centre for configurations without DCB estimation [latitude, longitude].
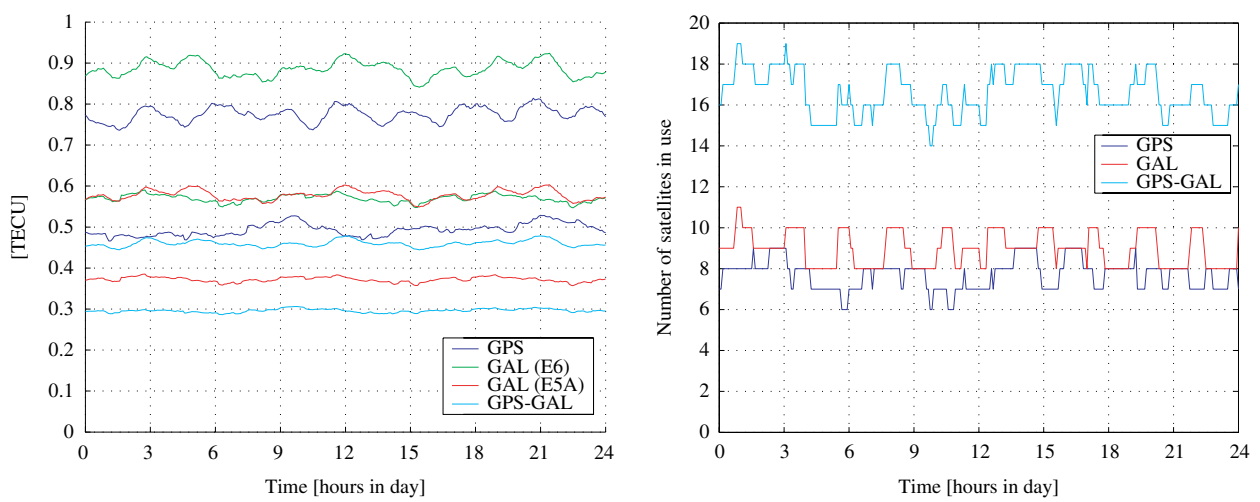

Figure 5. Time series of the standard deviation of the vertical TEC with different configurations at the origin, latitude-longitude [0,0] (left), and corresponding number of satellites in use (right). Two lines with the same colour: upper one is with DCB estimation, lower one is without. 